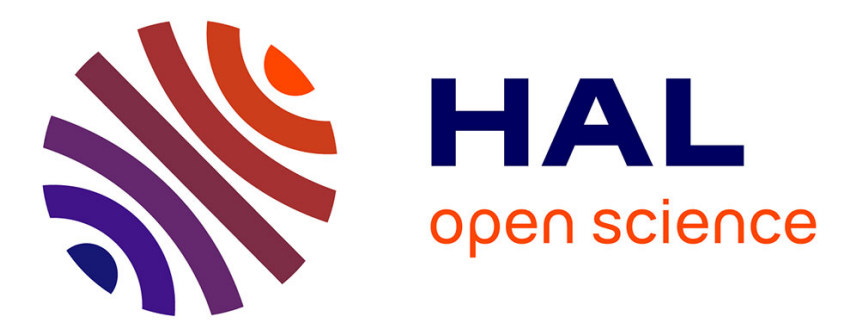

\title{
Dynamics of Drop Coalescence on a Surface: The Role of Initial Conditions and Surface Properties
}

\author{
R Narhe, D Beysens, Vadim Nikolayev
}

\section{To cite this version:}

R Narhe, D Beysens, Vadim Nikolayev. Dynamics of Drop Coalescence on a Surface: The Role of Initial Conditions and Surface Properties. International Journal of Thermophysics, 2005, 10.1007/s10765005-8593-4 . hal-01261693

\section{HAL Id: hal-01261693 \\ https://hal.science/hal-01261693}

Submitted on 26 Jan 2016

HAL is a multi-disciplinary open access archive for the deposit and dissemination of scientific research documents, whether they are published or not. The documents may come from teaching and research institutions in France or abroad, or from public or private research centers.
L'archive ouverte pluridisciplinaire HAL, est destinée au dépôt et à la diffusion de documents scientifiques de niveau recherche, publiés ou non, émanant des établissements d'enseignement et de recherche français ou étrangers, des laboratoires publics ou privés.

\section{(ㅇ)(1) $\$$}

Distributed under a Creative Commons Attribution - NonCommercial - NoDerivatives $\mid 4.0$ 
DYNAMICS OF DROP COALESCENCE ON A SURFACE: THE ROLE OF INITIAL CONDITIONS AND SURFACE PROPERTIES ${ }^{1}$

\author{
R. Narhe ${ }^{2}$, D. Beysens ${ }^{2,3}$ and V. Nikolayev ${ }^{2}$ \\ ESEME, SBT, CEA-Grenoble (France)
}
${ }^{2}$ Mailing address: CEA-ESEME, ESPCI-PMMH, 10 rue Vauquelin, 75231 Paris Cedex 5 - FRANCE

\footnotetext{
${ }^{1}$ Paper presented at the Fifteenth Symposium on Thermophysical Properties, June 22-27, 2003, Boulder, Colorado, U.S.A

${ }^{3}$ To whom correspondence should be addressed. E-mail: dbeysens@cea.fr
} 


\begin{abstract}
An investigation of the coalescence of two water drops on a surface is presented and compared with drop spreading. The associated capillary numbers are very low $\left(<10^{-5}\right)$. The drops relax exponentially towards equilibrium. The typical relaxation time $t_{\mathrm{c}}$ decreases with contact angle. $t_{\mathrm{c}}$ is proportional to the drop size $R$, thus defining a characteristic velocity $U^{*}=R / t_{c}$. The corresponding $U^{*}$ values are smaller by many orders of magnitude than the bulk hydrodynamic velocity $(U=\sigma / \eta$, with $\sigma$ the gasliquid surface tension and $\eta$ the viscosity). The dynamics of receding (coalescence) and spreading motion is found of the same order when coalescence or spreading is induced by a syringe. The dynamics of coalescence induced with the syringe deposition is systematically faster by an order of magnitude than condensation-induced coalescence. This disparity is explained by the coupling of the contact line motion with the oscillation of the drop observed for syringe deposition but absent for condensationinduced coalescence.
\end{abstract}

KEY WORDS: capillary; coalescence; contact angle; contact line velocity; wetting. 


\section{INTRODUCTION}

In industrial and material processing, the coalescence of drops plays very important role, for instance, liquid mixtures, polymers, sintering of alloys, etc. Apart from this, the study of the three phase contact line (i.e. the motion of the gas-liquid interface) dynamics during drop coalescence or spreading has attracted a number of scientists. The contact line dynamics is very sensitive to many factors, e.g. chemical defects, presence of liquid film on solid surface, the wetting properties of the substrate itself, etc. It is very difficult to characterize these factors in practice. The contact line motion along the solid surface for the case of partial wetting remains a very active field of study in spite of the numerous works which have been already published on this subject [1-7]. It was found that the contact line motion is incompatible with the standard hydrodynamic description where the no-slip boundary condition on the solid surface is enforced [1]. Such unusual properties of the fluid wedge cause slow response of the contact line to external influence in comparison to that of the bulk fluid. This slow response can be interpreted in terms of the anomalously high dissipation proper to the near contact line region of the liquid wedge. Still there is no certainty on the exact microscopic mechanism of the contact line motion. Some results are described satisfactory by one mechanism and some by others.

The dynamics of liquid spreading due to heterogeneities on solid surface have been studied in many cases. De Gennes [1] described the spreading of liquid in the presence of a precursor film. This precursor film facilitates the spreading and allows the hydrodynamics of spreading to be explained. However, the ellipsometric studies of Voué et al. [2] and Blake et al. [3] showed that even though the precursor film plays an important role during the spreading in the complete wetting regime, it is absent for the 
partial wetting regime which is confirmed by Ruijter et al. [4,5] analytically and experimentally. They found that the spreading for the partial wetting regime was much slower than the complete wetting case meaning that the application of de Gennes precursor film theory led to unphysical values for the parameters of the theory. Also three time dependent spreading dynamics, namely (a) the early time dynamics, (b) the intermediate time dynamics and (c) long time relaxation dynamics have been observed We conclude from these studies that in the quite common situation of partial wetting, the precursor film is absent and one should apply another model of contact line motion. The microscopic models proposed by Blake and Haynes [6] and Pomeau [7] take into account a phase transition in the immediate vicinity of the contact line. We note that most of them result in the following expression of the contact line velocity $v_{n}$ in the direction normal to the contact line as a function of the dynamic contact angle $\theta$ of the form,

$$
v_{n}=\frac{\sigma}{\xi}\left(\cos \theta_{e q}-\cos \theta\right)
$$

Here $\sigma$ is the surface tension, $\xi$ is the model-dependent parameter that we will call the "dissipation coefficient", and $\theta_{e q}$ is the equilibrium value of the contact angle. Another common feature of these theories is that they predict a large $\xi$ value with respect to $\eta$, the shear viscosity, so that the ratio

$$
K=\frac{\eta}{\xi}
$$

is smaller than unity. A small $K$ value means that the dissipation in the contact line region is large with respect to the dissipation in the bulk of the liquid. It was shown recently [14] that Equation (1) is valid for arbitrary drop surface if the slow drop dynamics is assumed. 
The purpose of the present article is (a) to extend the study of Andrieu et al. [8] and (b) to investigate several different ways to initiate the coalescence of the drops, essentially condensation growth and syringe deposition, thus evaluating the impact of the initial conditions (condensation chamber, syringe) on the dynamics of coalescence and spreading.

\section{EXPERIMENTAL}

For this study silicon wafer (untreated and treated) and polyethylene sheet $(\approx 50 \mu \mathrm{m}$ thick) were used as substrates with different average contact angles and hystereses. The coalescence of two drops is studied either in (a) a condensation chamber (chamber experiments, CE), where droplets grows by condensation and coalesce when they touch each other, and (b) by adding a small drop on the top of two neighboring drops (syringe experiments, SE). This method also enables (c) spreading of a single drop to be studied.

\subsection{Chamber Experiments}

The setup for the condensation experiment is typical for studying Breath Figures and has already been described in Ref. 8. Herein, we only outline some basic features. The condensation assembly consists of a Peltier-element thermostat (to lower the substrate temperature) in a closed Plexiglas chamber. Nitrogen gas saturated with water at room

temperature $\left(23^{\circ} \mathrm{C}\right)$ is sent into the chamber at a fixed flow rate $\left(0.6 \mathrm{~L} \cdot \mathrm{min}^{-1}\right)$. The experimental procedure consists of cooling the substrate to the desired temperature and sending $\mathrm{N}_{2}$ saturated with water vapor into the chamber. The growth of the condensation pattern is observed with an optical microscope and recorded with the CCD camera on videotapes. The video data are then analyzed by an image processing system. 


\subsection{Syringe Experiments}

In syringe experiments two small water drops of known volumes were deposited very close to each other on a substrate. In order to induce coalescence, a small drop is deposited on the top of one of the drops. The process of coalescence and relaxation was filmed with the CCD camera equipped with a macro-zoom lens and recorded on a video recorder. The initial process of fusion of two drops for both chamber and syringe experiments was observed with a high speed CCD camera (HCC1000 strobe, $1000 \mathrm{f} . \mathrm{s}^{-1}$ ). For spreading study, a small water drop of known volume was released on the substrate from a distance of 2 to $3 \mathrm{~mm}$. The syringe experiments were all performed at open room atmosphere (room temperature and humidity). In order to test a possible temperature and humidity influence, the syringe experiments were carried out at different temperatures of the substrate $T_{s}$ such that $T_{\mathrm{s}}>T_{\mathrm{D}}, T_{\mathrm{s}}=T_{\mathrm{D}}, T_{\mathrm{s}}=T_{\mathrm{D}}-5 \mathrm{~K}$, where $T_{\mathrm{D}}$ denotes the dew point temperature for the same ambient humidity. We observed that the change in temperature does not affect the relaxation of the composite drop. When $T_{\mathrm{s}}<$ $T_{\mathrm{D}}$, tiny condensing drops are visible on the substrate, some of them coalesce with the composite drop formed during the coalescence of two deposited drops. The volume change of the composite drop due to this multiple coalescences is negligible, and the triple line motion is not affected.

\subsection{Surface Properties}

The following substrates were used: (a) $50 \mu \mathrm{m}$ thick polyethylene sheet and (b) silicon wafers with different surface treatment. The surface properties, which determine the contact angle, are changed on the silicon surface using the silanization procedure as described in Ref. 9. However, in our case the substrate was kept above the cavity containing decyltrichlorosilane instead of its sidewise position as described in [9]. The 
contact angle is changed by varying the distance between substrate and the cavity while the silanization time was kept fixed (1 min). The contact angle of water on a substrate is measured by the sessile drop method. A small drop of $1 \mu \mathrm{L}$ is deposited on the substrate by means of a microliter syringe and observed using the CCD camera with a macro lens. The receding contact angle $\left(\theta_{\mathrm{r}}\right)$ and advancing contact angle $\left(\theta_{\mathrm{a}}\right)$ are measured by adding/removing a small amount of water to/from the drop with a micro syringe. The value of $\theta_{\mathrm{a}}$ and $\theta_{\mathrm{r}}$ for silicon and polyethylene substrates are given in Table I. Under $\theta_{\mathrm{eq}}$ we mean $\left(\theta_{\mathrm{a}}+\theta_{\mathrm{r}}\right) / 2$.

\section{RESULTS AND DISCUSSION}

\subsection{Coalescence in Condensation Chamber}

Figure 1 shows typical microscopic coalescence sequence for two hemispherical drops in condensation chamber. A new composite elongated drop is formed. Its shape can be characterized by the large axis $\left(2 R_{\mathrm{y}}\right)$ measured in the direction of the elongation and the small axis $\left(2 R_{\mathrm{x}}\right)$ measured in the perpendicular direction. The large and small drop radii $\left(R_{\mathrm{y}}\right.$ and $\left.R_{\mathrm{x}}\right)$ are defined as halfs of the corresponding axes. The composite drop finally becomes hemispherical of equilibrium radius $R$. The dynamics is very slow, and the complete relaxation takes a long time. The relaxation velocity is proportional to the restoring force $F$ which is defined by the change of the drop energy, i.e., the drop free surface area, see Nikolayev and Beysens [10]. The coalescence process is characterized by three time stages as observed by Andrieu et al. [8]: (a) formation of liquid bridge, (b) decrease of large radius $R_{\mathrm{y}}$ and increase of small radius $R_{\mathrm{x}}$ such that the ratio $R_{\mathrm{y}} / R_{\mathrm{x}}$ eventually reaches a value about unity, and (c) slow growth due to condensation.

Figure 2 shows the time evolution of large radius $R_{\mathrm{y}}$ and small radius $R_{\mathrm{x}}$ of composite drop. In stage 2 the restoring force $F_{y}$ can be approximated [5] by the expression, 


$$
F_{y}=2 \pi R_{y} \sigma_{l v}\left(\cos \theta-\cos \theta_{r}\right)
$$

where $R_{y}$ is time dependent drop radius and $\theta=\theta(t)$ is the time-dependent dynamic receding contact angle at points $M_{1}$ and $M_{2}$ that lie on the long axis. Equation (3) is exact for the spherical cap shape [4]. A rough evaluation of the initial value of this force per unit length,

$$
f=\frac{F_{y}}{2 \pi R_{y}}=\sigma_{l v}\left(\cos \theta_{0}-\cos \theta_{r}\right)
$$

can be obtained by estimating the initial (i.e. at the beginning of stage 2) contact angle $\theta_{0}=\theta\left(t=t_{0}\right)$, where $t_{0}$ is the initial time. To estimate this $\theta_{0}$ angle, we assume that during stage $1, R_{\mathrm{x}}$ does not change (its variation is indeed smaller than that of $R_{\mathrm{y}}$ ) because the contact line stays pinned. For estimation purposes it can be assumed that the composite drop at $t=0$ takes a spheroid shape which is described analytically by Nikolayev and Beysens [10]. By postulating $R_{\mathrm{y}}=2 R_{\mathrm{x}}$, at $t=t_{0}$, one can obtain a relationship between the composite drop volume $V_{\mathrm{c}}, R_{\mathrm{y}}$ and $\cos \theta_{0}$ as $V_{\mathrm{c}}=(\pi / 6) R_{\mathrm{y}}^{3}[(1-$ $\left.\cos \theta_{0}\right)^{1 / 2}\left(2+\cos \theta_{0}\right) /\left(1+\cos \theta_{0}\right)^{3 / 2}$ ] valid for a spheroidal drop [15]. The volume $V_{\mathrm{c}}$ is on the other hand is a sum of the volumes of two identical spherical-cap shaped drops of volume $V$ and radius $R_{l}, V_{\mathrm{c}}=2 V$. These drops are assumed to be at equilibrium (i.e. the contact angle is $\theta_{\mathrm{a}}$ after stage 3 where contact line advances very slowly due to condensation), just before coalescence begins. Their base radius $R_{l}$ can then be obtained from the expression [5]

$$
V=\frac{\pi}{3} R_{1}^{3} \frac{\left(1-\cos \theta_{a}\right)\left(2+\cos \theta_{a}\right)}{\left(1+\cos \theta_{a}\right) \sin \theta_{a}}
$$

Since the points $M_{1}$ and $M_{2}$ are assumed to be immobile during the first stage, $R_{\mathrm{y}}=2 R_{l}$. These equations allow $\theta_{0}$ and $f$ to be calculated, provided $\theta_{a}$ is given. The calculated $\theta_{0}$ 
values are reported in Table II. The value of $\theta_{0}$ is always markedly different from $\theta_{\mathrm{eq}}$, so that the contact angle hysteresis - which is a mean measure of the surface roughness does not influence much the force. Equation (4) can be compared to

$$
f \approx \sigma_{l v}\left(\cos \theta_{0}-\cos \theta_{e q}\right) \text {, }
$$

where the surface roughness influence is absent.

Generally speaking, the relaxation of an arbitrary physical quantity $R_{\mathrm{y}}$ (larger drop axis in our case) sufficiently close to its equilibrium value $R$, can be described by the equation

$$
\dot{R}_{y}=\frac{\left(R_{y}-R\right)}{t_{c}}
$$

which has the solution $R_{y}=R_{0} \exp \left[\left(t-t_{0}\right) / t_{c}\right]+R$, where $R_{0}$ is defined by the initial conditions. This suggests the exponential fit. The relaxation time $t_{\mathrm{c}}$ can be obtained by fitting of the relaxation data by the equation

$$
R_{x, y}(t)=R_{0} \exp \left[\frac{-\left(t-t_{0}\right)}{t_{c}}\right]+R+A\left(t-t_{0}\right) .
$$

The first term corresponds to the relaxation of the composite drop, which is dominating in regime 2; the third term approximately describes the slow growth due to the condensation which dominates regime 3 . The time of the beginning of coalescence is $t_{0}$. Its experimental value is imposed in the fit. $R_{0}, R, A$, and $t_{\mathrm{c}}$ are the fitting parameters. One can notice the good quality of this fit.

Figure 3 shows the relaxation time $t_{\mathrm{c}}$ versus the equilibrium drop size $R$ on a $\log -\log$ scale for the silicon I substrate. It should be noted that $t_{\mathrm{c}}$ should depend also on the difference between the sizes of two droplets. However, this dependence is very weak for small difference and the composite drop is nearly symmetric. For viscous relaxation 
driven by surface tension the relaxation time depends on $R$ and $U$ (where $U=\sigma / \eta$ ) value. In our case, the relaxation time $t_{\mathrm{c}}$ follows a linear variation with $R$ too;

$$
t_{c}=\left(\frac{1}{U^{*}}\right) R
$$

as shown in Fig.3.

The value of $U^{*}$ (slope of the $t_{\mathrm{c}}-R$ graph) characterizes the velocity of the contact line motion. This velocity is not to be confused with the contact line speed, which obviously varies with time during the relaxation process. The $U^{*}$ values obtained for silicon and polyethylene substrates are given in Table I, together with the treated quartz substrates used by Andrieu et al. It shows that $U^{*}$ for a silicon surface is one order of magnitude smaller than for polyethylene. The dynamics is faster for a larger contact angle (in polyethylene) as the leading capillary force that moves the contact line is larger, which is in good agreement with the theoretical [10] and numerical [14] predictions. According to them, $t_{\mathrm{c}}$ should vary with $\theta$ as

$$
t_{c}=\frac{1}{K} \frac{\eta}{\sigma} \phi(\theta) R
$$

This defines $U^{*}$ as

$$
U^{*}=K \frac{\sigma}{\eta} \frac{1}{\phi(\theta)}
$$

with [10]

$$
\phi(\theta)=45 \frac{1+\cos \theta}{\left(108+41 \cos \theta+14 \cos ^{2} \theta+17 \cos ^{3} \theta\right)(1-\cos \theta)}
$$

In Fig. 4 the values of $U^{*} / U$ are plotted with respect to $\theta_{e q}$ (with $U=$ $\sigma / \eta, \sigma=73 \mathrm{mN} . \mathrm{m}^{-1}, \eta=10^{-3}$ Pa.s.). Both condensation and syringe experiments were done many times and each data point was obtained by averaging over 15 to 20 
measurements. Although the data exhibit a large scatter, they can be reasonably fitted by the $K / \phi(\theta)$ variation (Fig. 4 , continuous curve), resulting in the value $K \approx 2.5 \times 10^{-6}$. The reasons of such a scatter cannot be found in the difference of restoring force due to different $\theta_{r}$. Indeed, in the framework of a linear approach (see e.g. [8-10] the magnitude of the restoring force cannot influence the relaxation time. The above scatter probably could be explained by the influence of defects. The effect of successive pinning and depinning of the contact line can result either in an increase of the relaxation time (the contact line stays longer on the defects) or to a decrease (the contact line jumps quickly between the defects). A linear approximation simulation [10] shows that these two antagonist effects nearly cancel. A non-linear approach [12] developed for a periodic defect pattern shows that the defects should slow the contact line motion. Their influence becomes stronger as the velocity of the contact line decreases.

\subsection{Spreading with Syringe}

Figure 5 shows $R$ vs $t$ data for the spreading of a water drop on a silicon surface in the syringe experiment. In this experiment a drop of known volume is deposited on a flat horizontal substrate surface and the complete process of spreading was recorded with video camera for drops of different size. The relaxation time is obtained by fitting the data with an exponential function. The relaxation time is comparable to the receding experiments with coalescence. It is interesting to make a comparison with the data of Rieutord et al. [13]. Fig. 5 shows that they can be fitted successfully to the same exponential relaxation. Both data exhibit comparable values of $U^{*}=3.65 \times 10^{-3} \mathrm{~m} . \mathrm{s}^{-1}$ and $11.75 \times 10^{-3} \mathrm{~m} . \mathrm{s}^{-1}$ (from the fits). Rieutord et al. have analytically proved the exponential behavior of the drop spreading when $\theta \rightarrow \theta_{e q}$. It is interesting to estimate the moving force in the spreading case and compare to the receding - controlled 
coalescence case. The volume $\left(V_{1}\right)$ of the added drop at the top of the other $\left(V_{2}\right)$ can be varied from $1 / 4$ to 1 volume ratio. The amplitude of the dynamic contact angle is less important and the effect of hysteresis is more pronounced as shown in Table II.

\section{CONCLUSION}

These experiments show that the dynamics of low viscous sessile drops during spreading and coalescence can be markedly affected by the initial conditions. We confirm and extend the data obtained by Andrieu et al. [8). The relaxation rate depends on the initial kinetic energy given to the drop at the beginning of the relaxation. The syringe deposition induces strong oscillations of the drop. At each oscillation, the drop surface "pulls" the contact line which accelerates its motion. In contrast, drop oscillations are not detected for the case of coalescence observed during the condensation and the relaxation turns out to be 10-100 times slower. This means that the contact line motion studies carried out with the traditional drop deposition method are not accurate enough because of uncontrollable oscillations, important especially for low viscosity fluids. The condensation-induced coalescence presents a more reliable way to study the contact line motion because of absence of any surface oscillations.

The slow contact line motion can be characterized by a "contact line dissipation" coefficient $\xi$, much larger than the dynamic viscosity $\eta$. It is very difficult to assign a precise value for the ratio $K=\eta / \xi$, which is found to be of the order $10^{-4}$ for the syringe deposition and $2.5 \times 10^{-6}$ in the condensation coalescence. Such small K values clearly show that the dynamics of low viscous sessile drops (spreading, coalescence) in the regime of partial wetting is limited by the dissipation at the region of the drop close to the contact line. This dissipation leads to relaxation 5 to 6 orders of magnitude slower 
than expected from bulk dissipation. Such a large $\xi$ value is expected within Pomeau theory [7] arguing that $K$ is the Arrhenius factor. However, in this theory, spreading is expected to be much faster than receding as the Arhenius factor is no longer present. We were unable to put in evidence such a difference in our experiments.

Alternatively, such a large $\xi$ value can be explained by the influence of the defects on the contact line motion discussed in [12]. It is shown in [12] that when the pulling force is small, the contact line motion is a sequence of "sticks" and "slips" that slows down the contact line and thus leads to a large effective value of $\xi$. However, the actual value of $\xi$ is much smaller. The relaxation rate increases with the contact angle, leading to an angle variation in reasonable agreement with the theory [10] of sessile drop relaxation confirmed by the recent numerical simulations [14].

\section{ACKNOWLEDGEMENTS}

We thank F. Rieutord for giving us his experiment data files and Laboratorie d'Electronique de Technologie de l'Information (LETI) at CEA- Grenoble for providing us silicon wafers. We are indebted to F. Palencia for helping us with video acquisition apparatus.

\section{REFERENCES}

1. De Gennes P.G, Rev. Mod. Phys. 57: 827 (1985).

2. M. Voué, M .P. Valignat, G. Oshanin, A.M. Cazabat, and J. de Coninck, Langmuir 14: 5951 (1998).

3. T.D. Blake, C. Decamps, J. De Coninck, M. De Ruijter, and M. Voué, Colloids and Surfaces A 154: 5 (1999). 
4. M. J. de Ruijter, J. De Coninck and G. Oshanin, Langmuir 15: 2209 (1999).

5. M.J. de Ruijter, J. De Coninck, T.D. Blake, A. Clarke and Rainkin, Langmuir 13: 7293 (1997).

6. T. D. Blake and J. M. Haynes, J. Colloid Interface Sci. 30: 421 (1969).

7. Y. Pomeau, C. R. Acad. Sci. IIb : Mec.,Phys .,Chim., Astron. 238: 411 (2000).

8. C. Andrieu, D. A. Beysens, V. S. Nikolayev, and Y. Pomeau, J. Fluid. Mech. 453: 427 (2002).

9. H. Zhao and D. Beysens, Langmuir 11: 627 (1995).

10. Vadim S. Nikolayev and Daniel A. Beysens, Phys. Rev. E 65: 46135 (2002).

11. V. S. Nikolayev and D. A. Beysens, Europhys. Lett. 64: 763 (2003).

12. V. S. Nikolayev J.Phys.: Cond. Mat. 17: 2111 (2005).

13. F. Rieutord, O. Rayssac, and H. Moriceau, Phys. Rev. E 62: 6861 (2000).

14. S. Iliev, N. Pesheva, and V. S. Nikolayev, Phys. Rev. E 72: 011606 (2005).

15. R. Narhe, D. Beysens and V.S. Nikolayev, Langmuir 20: 1213 (2004). 
Table I. The Contact Angles and Relaxation Rates of Composite Water Drop on Silicon with Various Treatment, Glass and Polyethylene Substrates.

\begin{tabular}{|c|l|l|c|l|l|l|}
\hline \multirow{2}{*}{ Substrate } & \multirow{2}{*}{$\theta_{r}\left({ }^{\circ}\right)$} & \multirow{2}{*}{$\theta_{a}\left({ }^{\circ}\right)$} & $\theta_{e q}\left({ }^{\circ}\right)$ & \multicolumn{2}{|c|}{ Coalescence } & Spreading \\
\cline { 5 - 7 } & & & $\begin{array}{c}\text { Chamber } \\
U^{*}\left(\mathrm{~m} . \mathrm{s}^{-1}\right)\end{array}$ & $\begin{array}{l}\text { Syringe } \\
U^{*}\left(\mathrm{~m}^{-1}\right)\end{array}$ & $\begin{array}{c}\text { Syringe } \\
U^{*}\left(\mathrm{~m} . \mathrm{s}^{-1}\right)\end{array}$ \\
\hline $\begin{array}{c}\text { Glass+silane } \\
{[8]}\end{array}$ & 46 & 60 & 53 & $(6.5 \pm 0.4) \times 10^{-6}$ & -- & -- \\
\cline { 2 - 7 } & 23 & 37 & 30 & $(1.2 \pm 0.1) \times 10^{-5}$ & -- & -- \\
\hline Silicon-I & $22 \pm 2$ & $25 \pm 2$ & 23.5 & $(2.5 \pm 0.12) \times 10^{-5}$ & $(1.89 \pm 0.12) \times 10^{-3}$ & $(1.19 \pm 0.04) \times 10^{-2}$ \\
\hline $\begin{array}{c}\text { Silicon-I } \\
+ \text { silane }\end{array}$ & $55 \pm 2$ & $79 \pm 2$ & 67 & $(1.47 \pm 0.19) \times 10^{-4}$ & $(3.4 \pm 0.65) \times 10^{-3}$ & $(9.3 \pm 1.6) \times 10^{-3}$ \\
\hline Silicon-II & $47 \pm 2$ & $57 \pm 2$ & 52 & $(9.74 \pm 0.12) \times 10^{-4}$ & $(9.54 \pm 0.7) \times 10^{-3}$ & $(9.3 \pm 1.6) \times 10^{-3}$ \\
\hline Silicon-III [13] & 10 & 12 & 11 & -- & -- & $3.65 \times 10^{-3}$ \\
\hline Polyethylene & $80 \pm 2$ & $90 \pm 2$ & 85 & $(7.24 \pm 0.7) \times 10^{-4}$ & $(6.15 \pm 0.6) \times 10^{-3}$ & $(4.1 \pm 0.9) \times 10^{-3}$ \\
\hline
\end{tabular}

Table II. Initial Contact Angle $\theta_{0}$ Calculated from Geometry and Capillary Force $f$ for Case of Coalescence and Spreading.

\begin{tabular}{|c|c|c|c|c|c|c|c|c|c|c|}
\hline \multirow[t]{4}{*}{ substrate } & \multirow{4}{*}{$\theta_{e q}\left({ }^{\circ}\right)$} & \multicolumn{4}{|c|}{$\begin{array}{c}\text { Condensation /deposition } \\
\text { coalescence }\end{array}$} & \multicolumn{5}{|c|}{ spreading } \\
\hline & & \multirow[t]{3}{*}{$\begin{array}{l}\theta_{0}\left({ }^{\circ}\right) \\
\text { calc. }\end{array}$} & \multirow[t]{3}{*}{$\theta_{r}\left(^{\circ}\right)$} & \multirow{2}{*}{\multicolumn{2}{|c|}{$f, \mathrm{mN} \cdot \mathrm{m}^{-1}$}} & \multirow{3}{*}{$\begin{array}{l}\theta_{0}\left({ }^{\circ}\right) \\
\text { calc. } \\
V_{1} / V_{2} \\
=1 / 4\end{array}$} & \multirow{3}{*}{$\begin{array}{c}\theta_{0}\left({ }^{\circ}\right) \\
\text { calc. } \\
V_{1} / V_{2} \\
=1\end{array}$} & \multirow{3}{*}{$\theta_{e q}^{\circ}=\theta_{a}$} & \multicolumn{2}{|c|}{$\begin{array}{l}f, \mathrm{mN}^{-1} \mathrm{~m}^{-1}, \\
\text { from Eq. (6) }\end{array}$} \\
\hline & & & & & & & & & $V_{1} / V_{2}$ & $V_{1} / V_{2}$ \\
\hline & & & & $\begin{array}{l}f \text { from } \\
\text { Eq.(4) }\end{array}$ & $\begin{array}{c}f \text {, from } \\
\text { Eq. }(6)\end{array}$ & & & & $=1 / 4$ & $=1$ \\
\hline \multirow[t]{2}{*}{ Glass + silane [8] } & 53 & 34.53 & 46 & 9.43 & 23.64 & -- & & -- & -- & -- \\
\hline & 30 & 19.51 & 23 & 1.61 & 10.51 & -- & & -- & -- & -- \\
\hline Silicon-I & 23.5 & 12.81 & 22 & 3.50 & 5.02 & 30.7 & 46.05 & 25 & 3.4 & 15.5 \\
\hline Silicon-I + silane & 67 & 50.42 & 55 & 4.64 & 32.58 & 88.0 & 104.7 & 79 & 11.3 & 32.5 \\
\hline Silicon-II & 52 & 32.35 & 47 & 11.88 & 21.91 & 66.3 & 85.68 & 57 & 10.4 & 34.3 \\
\hline Silicon-III [13] & 11 & -- & -- & -- & & -- & & -- & & -- \\
\hline Polyethylene & 85 & 61.59 & 80 & 22.06 & 34.73 & 98.1 & 113.1 & 90 & 10.3 & 28.6 \\
\hline
\end{tabular}




\section{FIGURE CAPTIONS}

Fig. 1. Photo of the coalescence process on $\mathrm{Si}$ (I) wafer in a condensation chamber. (i) $0.83 \mathrm{~s}$ (ii) $0.85 \mathrm{~s}$; (iii) $1.85 \mathrm{~s}$; (iv) $11.85 \mathrm{~s}$; (v) $26.85 \mathrm{~s}$; and (vi) $51.85 \mathrm{~s}$. The bar is of 50 $\mu \mathrm{m}$. The side of each photo corresponds to $172 \mu \mathrm{m}$.

Fig. 2. Time evolution of large radius $R_{\mathrm{y}}$ and small radius $R_{x}$ of composite drop formed due to coalescence of two water drops during chamber condensation on silicon I surface. The lines are the best fits to Eq.(8).

Fig. 3. Relaxation time $t_{\mathrm{c}}$ on Silicon I wafer by condensation in the chamber and in open room atmosphere (at $T_{R}=23^{\circ} \mathrm{C},>T_{\mathrm{D}}=18{ }^{\circ} \mathrm{C}$ ) with respect to the equilibrium size $R$ (log-log plot). Lines: fit to Eq. (9); full line $U^{*}=(3.0 \pm 0.15) \times 10^{-5} \mathrm{~m} \cdot \mathrm{s}^{-1}$; broken line: $U^{*}=(1.7 \pm 0.16) \times 10^{-5} \mathrm{~m} \cdot \mathrm{s}^{-1}$. The difference can be attributed to the evolution of the substrate cleanness.

Fig. 4. Experimental ratio $U^{*} / U$ from Table I with respect to $\theta_{e q}$ for different substrates and types of experiment (semi-log plot): full dots: chamber condensation; full curve: best fit to full dots to $K / \Phi(\theta)$, with $K=2.5 \times 10^{-6}$. Other symbols correspond to syringe experiments. Coalescence: inverted triangle, silicon I wafer; dot in open circle, silanized silicon I wafer; right triangle (left directed), silicon II wafer; right triangle (right directed), polyethylene. Spreading: plus sign in box, silicon I wafer; black slash in box, silanized silicon I wafer; open circle, silicon II wafer; dot in open box, silicon III wafer (the data from Ref. 13); times sign in box, polyethylene. $\left(U=\sigma / \eta=73 \mathrm{~m} \cdot \mathrm{s}^{-1}\right)$

Fig. 5. $R(t)$ data for spreading of water drop on silicon I surface in the syringe experiment (circles) and the data of Rieutord et al.[13] (squares). The fit to the exponential relaxation and the resulting $U^{*}$ values are shown too. For syringe experiment (circle), $V_{1} / V_{2}=1 / 4$. 


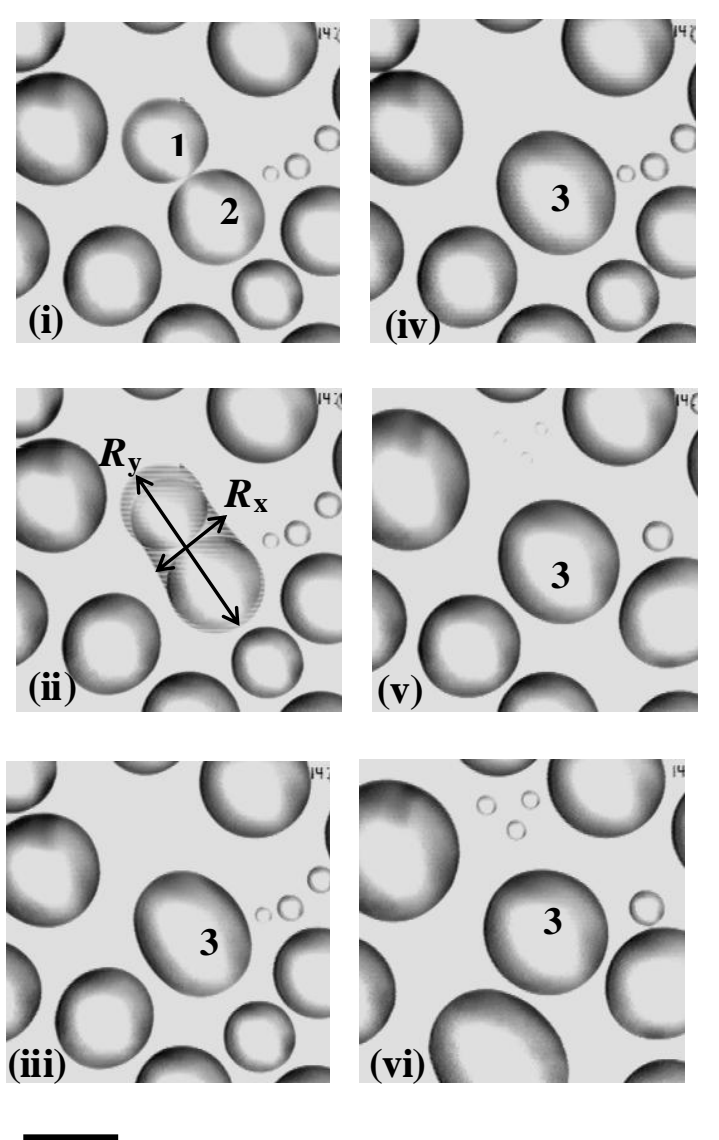

Fig.1 


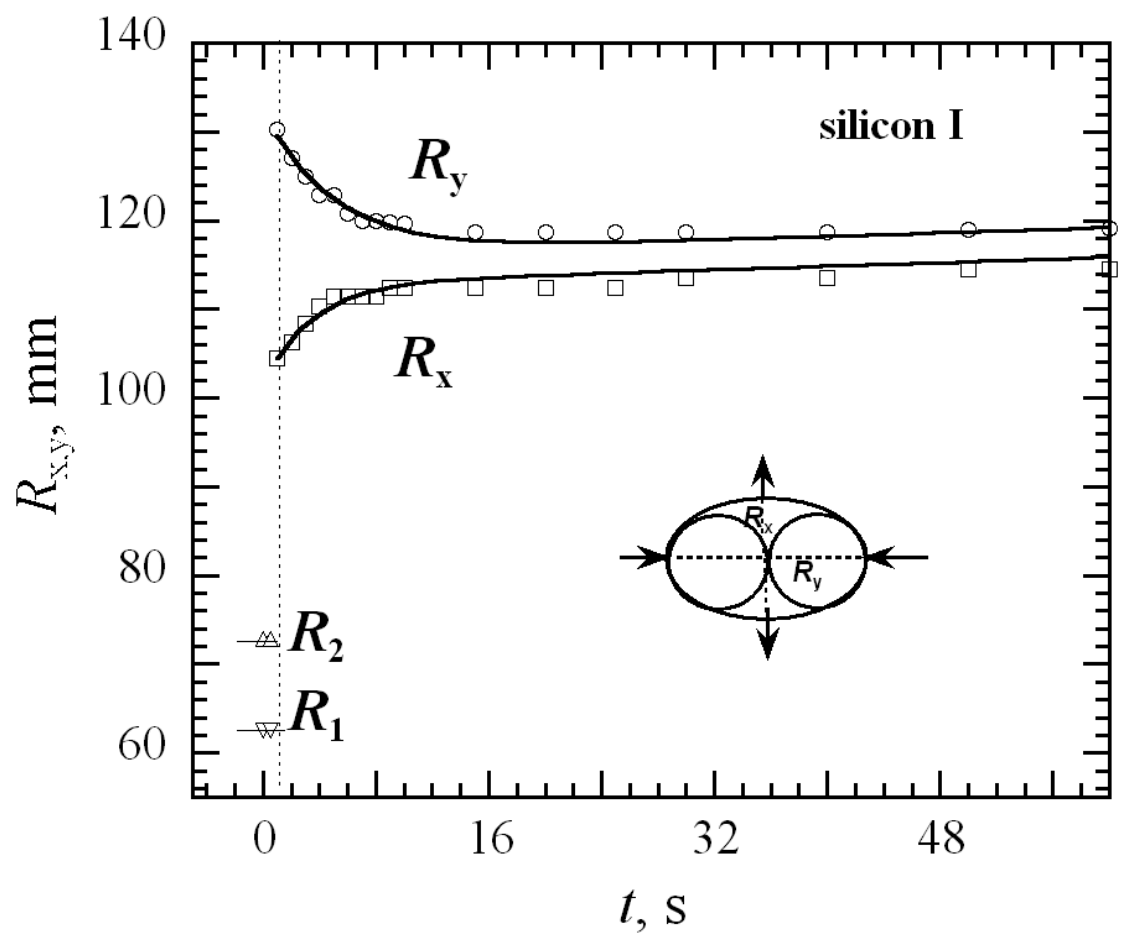

Fig.2 


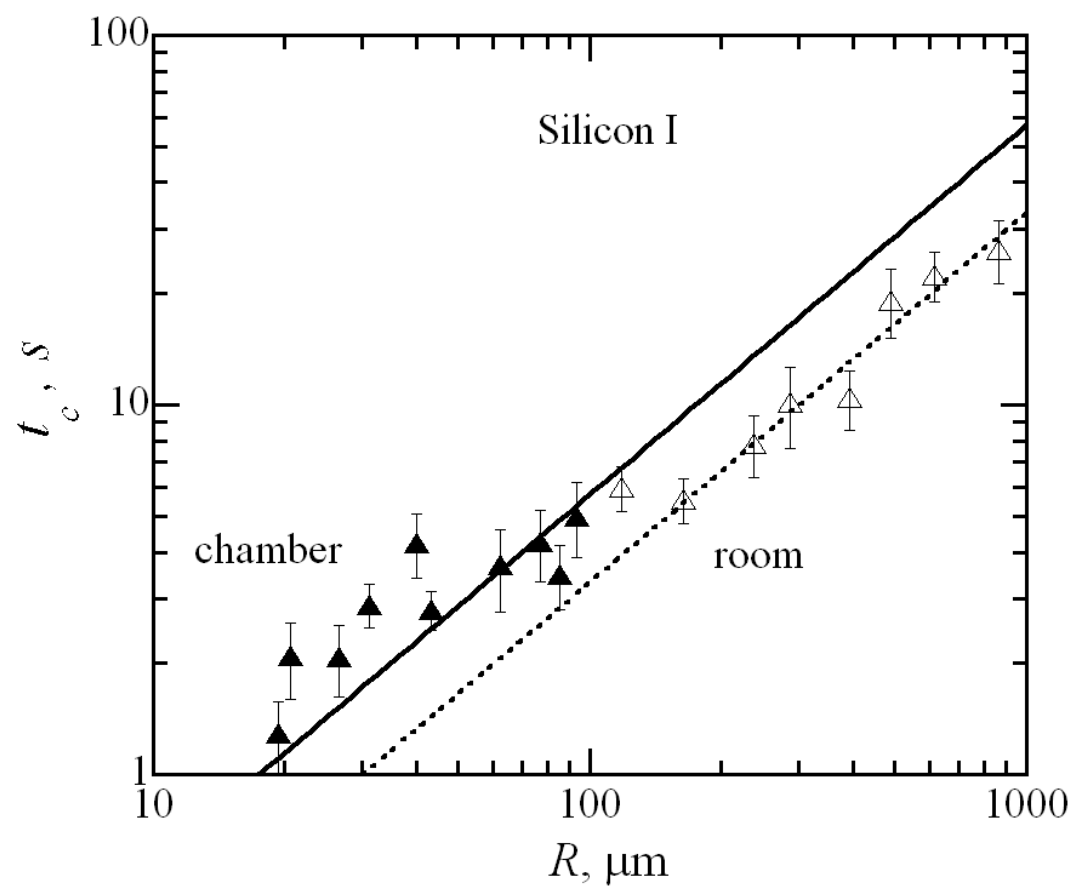

Fig.3. 


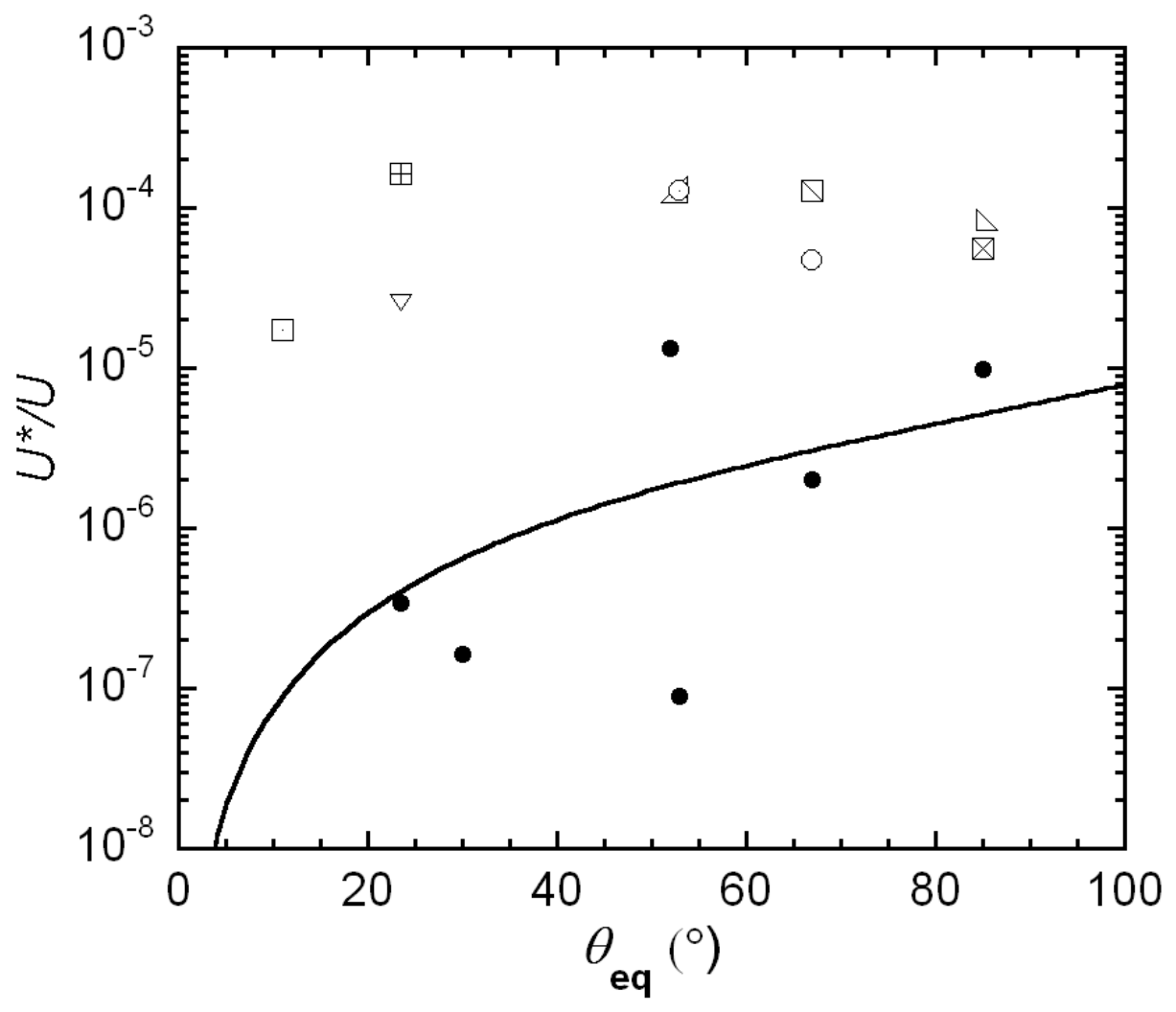

Fig. 4. 


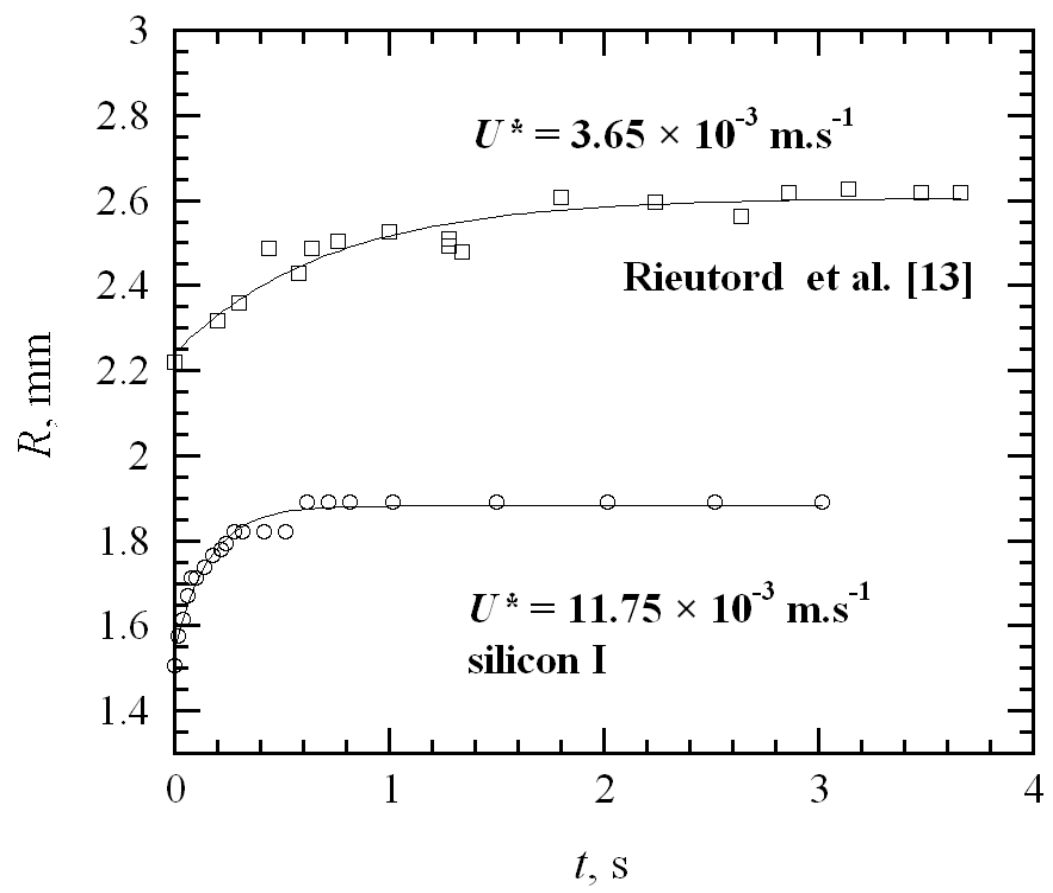

Fig. 5. 